\title{
openheart Case of placental insufficiency and premature delivery in a Fontan pregnancy: physiological insights and considerations on risk stratification
}

\author{
Maria Victoria Ordoñez (D) , ', Giovanni Biglino (D) , ${ }^{1,2,3}$ Massimo Caputo, ${ }^{1,2}$ \\ Brenda Kelly, ${ }^{4}$ Aarthi Mohan, ${ }^{5}$ Johanna Trinder, ${ }^{5}$ Stephanie L Curtis ${ }^{1}$
}

To cite: Ordoñez MV, Biglino G, Caputo M, et al. Case of placental insufficiency and premature delivery in a Fontan pregnancy: physiological insights and considerations on risk stratification. Open Heart 2021;8:e001211. doi:10.1136/ openhrt-2019-001211

Received 27 February 2020 Revised 26 September 2020 Accepted 2 October 2020
Check for updates

(c) Author(s) (or their employer(s)) 2021. Re-use permitted under CC BY-NC. No commercial re-use. See rights and permissions. Published by BMJ.

${ }^{1}$ University Hospitals Bristol and Weston NHS Foundation Trust, Bristol, UK

${ }^{2}$ Bristol Medical School, University of Bristol, Bristol, UK ${ }^{3}$ National Heart and Lung Institute, Imperial College London, London, UK

${ }^{4}$ Guy's King's and Saint Thomas' School of Medicine, London, UK ${ }^{5}$ Obstetrics, St Michael's Hospital Bristol, Bristol, UK

Correspondence to Dr Maria Victoria Ordoñez; victoria.ordonez@uhbw.nhs.uk

\section{ABSTRACT}

Objectives The coexistence of two complex physiologies such as Fontan and pregnancy is still not fully understood. We aim to add a unique and essential knowledge to help our colleagues in the management of Fontan patients that undergo pregnancy as well as the fetus and the placenta perfusion.

Methods and results We analyse the coexistence of Fontan and pregnancy physiology on a complex case of a woman with hypoplastic left heart syndrome palliated with a univentricular repair who became pregnant, delivered very prematurely and had atypical placental findings.

Conclusion Histopathological analysis of the placenta could help us to refine the understanding of Fontan physiology adaptation during pregnancy, predict women and fetal outcomes as well as to plan a better prepregnancy status. However, further evidence is needed in order to reach a more solid and unified conclusion.

\section{INTRODUCTION}

An understanding of the interaction between pregnancy physiology and Fontan physiology is crucial when predicting maternal and fetal outcomes for these women embarking on pregnancy. Maternal haemodynamic adaptation is limited in women with univentricular physiology as they are unable to increase cardiac output to meet the increasing physiological demands of pregnancy. Poor placental health has been previously described in patients with a Fontan circulation with increased fibrin deposition being the universal histopathological feature. ${ }^{1}$ Here we analyse the coexistence of these two physiologies in a complex case of a woman with hypoplastic left heart syndrome (HLHS) palliated with a univentricular repair who became pregnant, delivered very prematurely and had atypical placental findings.

\section{Key questions}

What is already known about this subject?

- Unfortunately, no evidence is available regarding placentation and its development during pregnancy in a patient with univentricular physiology. This interesting investigation describes current challenge situations that not only adult congenital heart disease cardiologists, but also obstetrician and fetal specialists presently face in patients with Fontan palliation that undergo pregnancy.

What does this study add?

- The strengths provided on our manuscript are the thorough and multidisciplinary approach that these patients warrant throughout their pregnancy as well as the importance of the pathological analysis of the placenta which is paramount in order to have a better physiology understanding and its implications.

How might this impact on clinical practice?

- We strongly believe that our manuscript adds a very valuable and unique experience and knowledge that would definitely be very helpful for our colleagues in the management of Fontan patients that undergo pregnancy as well as the fetus and the placenta perfusion.

- In the final analysis, the paper creates a paradigm for future studies of the evolution of essential guidelines and protocols tailored to these subsets of patients with a more global understanding of the placenta development, its consequences, different patterns of presentation and what risk this imposes pregnancy to the Fontan palliation.

\section{METHODS}

A 23-year-old patient with HLHS and mitral atresia and who had undergone a Norwood operation and extracardiac Fontan procedure. Her pre-pregnancy weight was $64 \mathrm{~kg}$ and she had no peripheral oedema. Fetal ultrasound confirmed a viable intrauterine singleton pregnancy.

A liver fibroscan performed before pregnancy showed a stiffness score $>17 \mathrm{KPa}$, 
indicating Fontan-related hepatic congestion. She also had a raised alanine aminotransferase of $125 \mathrm{U} / \mathrm{L}$. She was taking warfarin, as is our practice in all Fontan patients, and this was changed to full anticoagulation dose low molecular weight heparin.

An echocardiogram performed prior to pregnancy showed normal single right ventricular (RV) function with a tissue Doppler imaging peak systolic wave (TDI $\mathrm{S}^{\prime}$ wave) of $9 \mathrm{~cm} / \mathrm{s}$ at the base of the anterior RV wall, tricuspid annular plane septal excursion (TAPSE) of $1.9 \mathrm{~cm}$ and low velocity venous flow. There was mild atrioventricular $(\mathrm{AV})$ valve regurgitation and no outflow tract obstruction.

Cardiac MRI (CMRI) pre-pregnancy showed mildly impaired single ventricular (RV) function (ejection fraction $43 \%$ ), an unobstructed Fontan circuit and mild AV valve regurgitation (regurgitant fraction 3\%). In addition, the pulmonary arteries were slightly hypoplastic with the left being moreso than the right. Ostial left pulmonary artery (LPA) was $8 \mathrm{~mm}$ and mid-vessel $9 \mathrm{~mm}$, compared with a mid-right pulmonary artery measurement of $13 \mathrm{~mm}$. Pulmonary perfusion was normal and there were no signs of flow acceleration at rest.

Routine cardiac catheterisation 4 years prior to pregnancy showed unobstructed Fontan pathways with no pressure gradients but anatomically mild proximal LPA narrowing measuring $8 \times 9 \mathrm{~mm}$ compared with $12 \times 10 \mathrm{~mm}$ distally. Fontan pressure was $12 \mathrm{~mm} \mathrm{Hg}$. End-diastolic pressure was not measured at that time.

\section{RESULTS}

The patient was first seen in the multidisciplinary obstetric cardiac clinic at 6 weeks' gestation. This was her first pregnancy.

At that time, she was asymptomatic from a cardiovascular point of view, with an unremarkable clinical examination: blood pressure (BP) was $110 / 60 \mathrm{~mm} \mathrm{Hg}$, heart rate 70 beats/min, oxygen saturations $96 \%$ on air, single S1 and S2, and a pansystolic murmur loudest at the left sternal edge.

During pregnancy, she was seen regularly every 3 weeks at our tertiary centre high-risk cardiac antenatal clinic. She continued to be well, with oxygen saturations 94\%-96\% and BP 110/60-119/75 mm Hg. No clinical or electrocardiographic signs of abnormal rhythms were noted, nor any symptoms of heart failure.

With relation to fetal well-being, she experienced a selflimiting episode of fresh vaginal bleeding at 12 weeks. A pregnancy scan at 15 weeks' gestation showed a large intrauterine haematoma/area of haemorrhage, measuring $59 \times 55 \times 22 \mathrm{~mm}$ just superior to the cervix. Fetal growth was normal. After discussion with the woman regarding risks and benefits, a decision was made to continue with full-dose low molecular weight heparin.

At 19, 21 and 24 weeks' gestation, further fetal ultrasound was performed (figure 1). The placenta had an abnormal appearance with sonographic features to
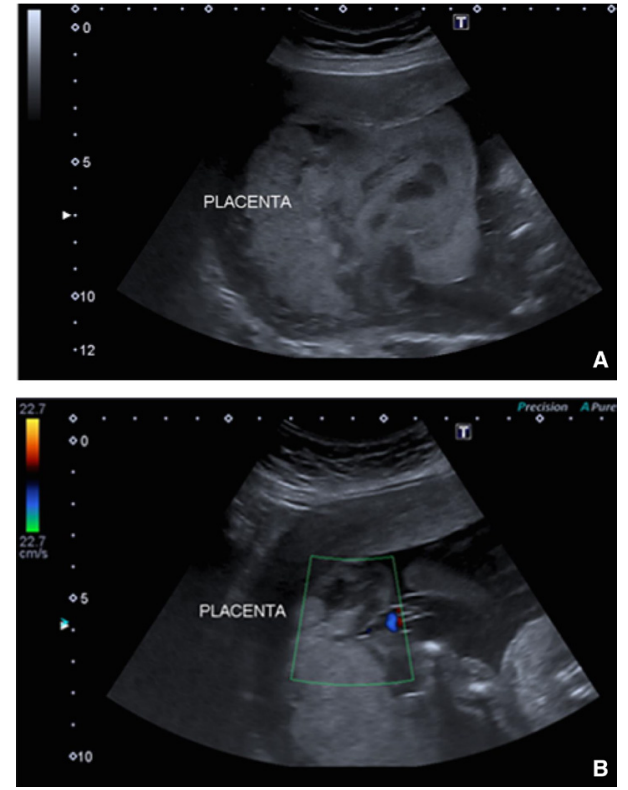

Figure 1 Fetal ultrasound images at week 19 (A) and 21 (B). (A) 2D image showing a dysplastic and enlarged placenta with possible fetal growth restriction. (B) Colour image showing placental perfusion.

suggest areas of placental infarction. Although fetal growth and umbilical artery Dopplers remained normal, she was counselled by a fetal medicine specialist that such appearances were associated with a high risk of, not only fetal growth restriction, but also an acute placental event such as a placental abruption and possible in utero fetal demise. The maternal echocardiogram at that time showed no changes in the function of the single ventricle (TAPSE $17 \mathrm{~mm}$ and basal anterior RV TDI S' wave 8 $\mathrm{cm} / \mathrm{s}$ ) without progression of $\mathrm{AV}$ valve regurgitation but a mild stenosis at the ostium of the LPA, which had not previously been seen on echo, though noted on CMRI. Flow in the Fontan circuit was appropriately low velocity.

At week 25, she experienced spontaneous rupture of membranes. She presented to hospital 6 hours later in advanced labour and spontaneously delivered a female infant weighing $700 \mathrm{~g}$ (25th centile) who was transferred to the neonatal intensive care unit. The baby died at 6 days of age due to necrotising enterocolitis and extreme prematurity.

This mother remained, apart from transient 20-30 min of dyspnoea shortly after delivery, cardiovascularly well during labour, delivery and the postnatal period with no clinical signs or symptoms of infection.

She was discharged on postnatal day 6 with normal BP $(115 / 67 \mathrm{~mm} \mathrm{Hg})$, no postpartum complications and no cardiovascular symptoms. An echocardiogram performed on postnatal day 13 revealed progression of the $\mathrm{AV}$ valve regurgitation to a moderate level, with stable single ventricular function, normal Fontan flow and less high velocity flow at the ostium of the LPA. The latter feature was interpreted as normal in the context of highvolume load during pregnancy and post partum. 

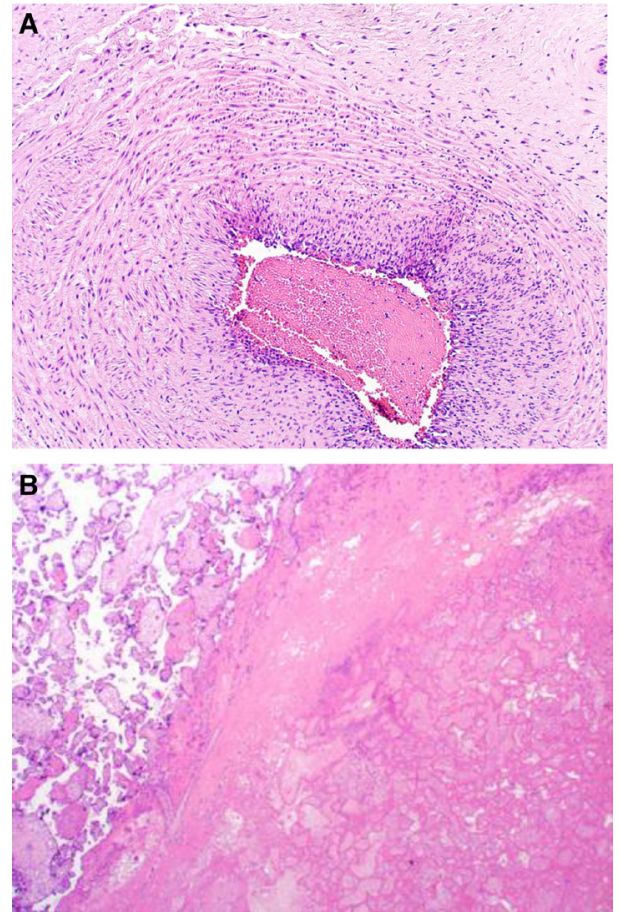

Figure 2 Placenta histology image of the umbilical cord showing the chorionic vasculitis $(A)$ and the infarction of the chorionic villi (B).

Placental histology showed maturation of the chorionic villi appropriate for the stage of gestation with evidence of acute maternal and fetal inflammatory response, chorionic vasculitis, funisitis and acute umbilical cord vasculitis, involving two out of the three vessels. These findings were suggestive of acute chorioamnionitis. In addition to this, there was a related area of parabasal infarction of chorionic villi with decidual haematoma, presumably secondary to placental abruption. There was no increased fibrin deposition (figure 2).

\section{DISCUSSION}

Fetal outcomes have been reported to be poor in women with a Fontan circulation undergoing pregnancy, with rates of miscarriage and intrauterine growth restriction (IUGR) in excess of $50 \%$, and prematurity in excess of $75 \% .^{2}$ An understanding of the complex interplay between Fontan physiology, the maternal haemodynamic adaptation to pregnancy and impact on fetoplacental circulation is pivotal for predicting potential maternal and fetal complications in single-ventricle patients.

Fontan completion entails offloading the single ventricle and improving oxygenation at the expense of a high systemic venous pressure and low cardiac output $(\mathrm{CO}){ }^{3}$ These patients have limited reserve to increase their CO to match physiological needs. ${ }^{45}$

During normal pregnancy, there is an increase in stroke volume and heart rate, and a decrease in systemic vascular resistance. In a Fontan patient, these haemodynamic changes are not well tolerated due to the absence of a subpulmonary ventricle to pump venous blood into the pulmonary circulation and a higher pulmonary vascular resistance. ${ }^{3}$ Therefore, an increase in the systemic venous pressure and lower CO secondary to a curtailed preload are expected. The impact of the Fontan circulatory haemodynamics in pregnancy on placental perfusion is inadequately understood. It is possible that the inability of the Fontan physiology to augment the stroke volume during pregnancy, combined with the increase in systemic venous pressure, may lead to placental vascular dysfunction, hypoperfusion and infarction or abruption.

Fontan-associated liver disease is a well-described consequence of the palliated single ventricle circulation and occurs as a consequence of chronic venous hypertension. ${ }^{6}$ As with the liver, the placenta seems to represent another site of end-organ damage secondary to the abnormal haemodynamics of the Fontan and its response to the changes in pregnancy. ${ }^{7}$

Despite literature and clinical experience suggesting poor perinatal outcomes, there is a dearth of literature on the fetoplacental unit in these women with, to our knowledge, only one small case series reported so far. ${ }^{1}$ Authors described detailed histology of the placentas from 13 deliveries in 7 women with Fontan circulation. Only 2 out 13 deliveries were at term with remaining pregnancies complicated by pre-eclampsia, preterm rupture of membranes, IUGR and placental abruption. Although there was wide variation in placental weight and villous maturity (likely reflecting range of gestational age of delivery), the striking feature common to all placentas examined was excessive subchorionic fibrin deposition. ${ }^{1}$ Authors speculated that excessive fibrin deposition may be due to 'stagnation of placental blood flow', namely venous stasis secondary to elevated venous resistance, and suggested that this could impair placental perfusion. While this is an interesting hypothesis, the nature of the study design means that selection bias cannot be discounted. While perivillous and intervillous fibrin deposition is associated with placental dysfunction in conditions such as pre-eclampsia and chorionic histiocytic intervillositis, subchorionic fibrin deposition is not infrequently seen in placentas subjected for routine examination and may be a non-specific finding. Moreover, the histological findings reported in our case suggest that poor outcome in the absence of excessive subchorionic fibrin deposition is possible. It may be that placental venous stasis occurs but fibrin deposition does not appear to be a necessary finding or event contributing to placental dysfunction in this high-risk group of women.

In our patient, evidence of a placental bleed and subsequently placental infarcts were apparent on scan from early second trimester in spite of relatively stable maternal cardiovascular status. Umbilical artery Doppler abnormality is an accepted marker of placental insufficiency. What is surprising in the case presented, is that despite sonographic placental findings, both fetal growth and umbilical artery Dopplers appeared normal. This is in contrast to our previously reported case of poor fetal outcome in a woman with good Fontan haemodynamics 
but whose fetus suffered severe IUGR. ${ }^{8}$ No placental pathology was available but the gross appearances on ultrasound were similar to those presented here and in the recent Mayo Clinic series. ${ }^{1}$

The current challenge for those caring for women with Fontan is that we have little evidence base from which to give individualised advice to prospective mothers, other than telling them that pregnancy carries with it a high chance of miscarriage or life-changing prematurity. The effects of cyanosis are well documented, but we do not know the effect of low cardiac output and chronic venous hypertension on the growing fetus. Anticoagulation is an added and unpredictable additional risk and the benefits of it are not well described in the pregnant Fontan, yet it significantly increases the risk of bleeding in pregnancy and adds to the already elevated fetal risk. ${ }^{9} 10$ Though some centres only fully anticoagulate Fontan patients with risk factors, such as an existing right to left shunt, reduced ventricular function or history of arrhythmia, it is our practice to anticoagulate all of these patients.

In this case it may have resulted in the placental bleeding, and does raise the question of whether or not full anticoagulation should be reconsidered in these women during pregnancy. It may be that antiplatelet therapy and/or prophylactic dose low molecular weight heparin might be more prudent. There are little data on this in the literature as most of the evidence relating to anticoagulation in pregnant patients with cardiac problems pertains to women with mechanical valves, an entirely different cohort of patients.

Fetal loss in the Fontan remains unpredictable. The additional challenge also lies in caring for such women in a subsequent pregnancy after previous adverse outcome when standard maternal, fetal and placental well-being surveillance does not predict outcome.

Given the rarity of the condition, there needs to be greater international collaboration between cardiologists, obstetricians and pathologists to more comprehensively study these issues. Setting up registries and databases so that these patients can be prospectively studied in order to best improve outcomes for mother and baby would be an important first step. Additionally, computational models of the circulation (including the uterine circulation) hold promise for carrying out parametric studies and generate insights into the complex interplay of various haemodynamic factors. ${ }^{11} 12$

\section{CONCLUSION}

Placenta pathology analysis should not be overlooked in these subsets of patients as it adds essential information about the adaptation of the univentricular physiology to pregnancy. Further analysis is needed in order to create a pre-pregnancy risk stratification score which will help us to improve pre-pregnancy clinical status.

Acknowledgements The authors thank Dr Andrew Bamber for providing the placenta histology images. The authors also acknowledge the generous support of the Grand Appeal (Bristol Royal Hospital for Children Charity) and the British Heart Foundation.

Contributors MV0-writing process, research on the field, coordination and submission. GB-writing process and editing. MC-writing and editing process. JT, $\mathrm{BK}$ and $\mathrm{AM}$ - writing of the obstetric segment and care of the patient. SLC—lead of the case report, writing and editing process.

Funding The authors have not declared a specific grant for this research from any funding agency in the public, commercial or not-for-profit sectors.

Competing interests None declared.

Patient consent for publication Obtained.

Provenance and peer review Not commissioned; externally peer reviewed.

Data availability statement All data relevant to the study are included in the article or uploaded as supplemental information. Data were obtained from medical records under patient's permission.

Open access This is an open access article distributed in accordance with the Creative Commons Attribution Non Commercial (CC BY-NC 4.0) license, which permits others to distribute, remix, adapt, build upon this work non-commercially, and license their derivative works on different terms, provided the original work is properly cited, appropriate credit is given, any changes made indicated, and the use is non-commercial. See: http://creativecommons.org/licenses/by-nc/4.0/.

\section{ORCID iDs}

Maria Victoria Ordoñez http://orcid.org/0000-0001-6474-7013

Giovanni Biglino http://orcid.org/0000-0003-0413-149X

\section{REFERENCES}

1 Phillips AL, Cetta F, Kerr SE, et al. The placenta: a site of endorgan damage after Fontan operation. A case series. Int J Cardiol 2019;289:52-5

2 Cauldwell M, Steer PJ, Bonner S, et al. Retrospective UK multicentre study of the pregnancy outcomes of women with a Fontan repair. Heart 2018;104:401-6.

3 Gewillig M, Brown SC. The Fontan circulation after 45 years: update in physiology. Heart 2016;102:1081-6.

4 Bukowski R, Hansen NI, Pinar H, et al. Altered fetal growth, placental abnormalities, and stillbirth. PLoS One 2017;12:e0182874.

5 Garcia Ropero A, Baskar S, Roos Hesselink JW, et al. Pregnancy in women with a Fontan circulation: a systematic review of the literature. Circ Cardiovasc Qual Outcomes 2018;11:e004575.

6 Daniels CJ, Bradley EA, Landzberg MJ, et al. Fontan-Associated Liver Disease: Proceedings from the American College of Cardiology Stakeholders Meeting, October 1 to 2, 2015, Washington DC. J Am Coll Cardiol 2017;70:3173-94.

7 Mikolasevic I, Filipec-Kanizaj T, Jakopcic I, et al. Liver disease during pregnancy: a challenging clinical issue. Med Sci Monit 2018;24:4080-90.

8 Ordonez MV, Trinder J, Curtis SL. Success in a Fontan pregnancy: how important is ventricular function? Cardiol Young 2019;29:225-7.

9 Steinberg ZL, Dominguez-Islas CP, Otto CM, et al. Maternal and Fetal Outcomes of Anticoagulation in Pregnant Women With Mechanical Heart Valves. J Am Coll Cardiol 2017;69:2681-91.

10 Silversides CK, Grewal J, Mason J, et al. Pregnancy outcomes in women with heart disease: the CARPREG II study. J Am Coll Cardiol 2018;71:2419-30.

11 Corsini C, Cervi E, Migliavacca F, et al. Mathematical modelling of the maternal cardiovascular system in the three stages of pregnancy. Med Eng Phys 2017;47:55-63.

12 Carson J, Lewis M, Rassi D, et al. A data-driven model to study utero-ovarian blood flow physiology during pregnancy. Biomech Model Mechanobiol 2019;18:1155-76. 